\title{
Chemotherapy-Induced Peripheral Neurotoxicity in Cancer Survivors: Predictors of Long-Term Patient Outcomes
}

\author{
Eva Battaglini, $\mathrm{PhD}^{1}$; David Goldstein, $\mathrm{MBBS}^{1}$; Peter Grimison, PhD, MBBS ${ }^{2,3}$; Susan McCullough ${ }^{4}$; \\ Phil Mendoza-Jones ${ }^{4}$; and Susanna B. Park, $\mathrm{PhD}^{5}$
}

\begin{abstract}
Background: Chemotherapy-induced peripheral neurotoxicity (CIPN) is a major adverse effect of cancer treatment. However, its impact remains poorly understood. This study aimed to investigate the impact associated with CIPN on the lives of cancer survivors. Patients and Methods: A volunteer sample of 986 individuals who had received neurotoxic chemotherapy completed an anonymous, cross-sectional survey. Outcomes assessed included CIPN symptoms, pain, neuropathic pain, quality of life (QoL), physical activity, and comorbid health conditions via the Self-Administered Comorbidity Questionnaire. Results: Respondents had a mean age of 58 years (SD, 10.7), and $83.2 \%$ were female. Most were treated for breast (58.9\%) or colorectal cancer (13.5\%); had received docetaxel (32.7\%), paclitaxel $(31.6 \%)$, or oxaliplatin $(12.5 \%)$; and had completed treatment $3.6 \pm 3.5$ years previously. We found that $76.5 \%$ of respondents reported current CIPN. Respondents reporting severe CIPN had poorer QoL, more comorbidities, and higher body mass index, and more often received multiple neurotoxic chemotherapies than those with mild CIPN. Respondents who completed the survey $\leq 1$ year after completing chemotherapy did not differ in reported CIPN or pain compared with respondents who completed chemotherapy $\geq 6$ years earlier. However, respondents who completed chemotherapy $\geq 6$ years earlier reported better QoL. Multivariable linear regression analyses revealed predictors of CIPN severity as follows: $F(7$, $874)=64.67 ; P<.001 ; R^{2}=0.34$, including pain $(\beta=-0.36 ; P<.001)$, burning pain $(\beta=0.25 ; P<.001)$, sex (male sex associated with greater CIPN: $\beta=0.14 ; P<.001$ ), years since completing chemotherapy (shorter time associated with greater CIPN; $\beta=-0.10 ; P<.001$ ), age $(\beta=0.80 ; P=.006)$, number of comorbid conditions $(\beta=0.07$; $P=.02)$, and body mass index $(\beta=0.07 ; P=.02)$. Conclusions: Respondents with a high CIPN symptom burden experienced poorer general health and QoL. Improvements in CIPN may be more likely soon after treatment. However, improvements in QoL may occur over time in those with chronic symptoms. CIPN seems to have lasting impacts on cancer survivors, and understanding risk factors is important to enable the design of further preventive and therapeutic management strategies.
\end{abstract}

J Natl Compr Canc Netw 2021;19(7):821-828 doi: 10.6004/jnccn.2021.7026

\footnotetext{
${ }^{1}$ Prince of Wales Clinical School, University of New South Wales Medicine, University of New South Wales, Kensington; ${ }^{2}$ The Chris O'Brien Lifehouse, Camperdown; ${ }^{3}$ Sydney Medical School, Faculty of Medicine, University of Sydney, Sydney; ${ }^{4}$ Translational Cancer Research Network Consumer Advisory Panel, Sydney; and ${ }^{5}$ Brain and Mind Centre, Faculty of Medicine and Health, University of Sydney, Camperdown, Australia.
}

\section{Background}

Chemotherapy-induced peripheral neurotoxicity (CIPN) is a major adverse effect (AE) of cancer treatment that can lead to early treatment cessation, lasting symptoms, and functional difficulties. Commonly used chemotherapies, including taxanes, platinum compounds, vinca alkaloids, bortezomib, and thalidomide, produce CIPN in a significant proportion of patients. With advances in diagnosis and treatment, increasing numbers of patients experience long-term survival. ${ }^{1}$ These improvements accompany an increased need to understand the impact of neurotoxic chemotherapies on cancer survivors' lives.

CIPN is characterized by tingling, numbness, and pain in the extremities of the limbs, leading to problems with walking, balance, and fine motor function. ${ }^{2}$ It is a major complaint across a range of cancers and neurotoxic agents, ${ }^{3-6}$ with a significant impact on quality of life (QoL). ${ }^{3,78}$ Although studies have investigated the development of chronic CIPN symptoms and their impact over time, ${ }^{9-12}$ there is a lack of complete understanding regarding the trajectory of CIPN over the long term and the lasting impact this has on cancer survivors.

This large-scale survey focused on CIPN as a key AE of neurotoxic chemotherapy, using validated measures to assess CIPN, QoL, and physical activity. We aimed to investigate the impacts associated with CIPN symptom burden on the lives of cancer survivors across a range of domains, examining symptoms over time and interactions with other treatment AEs, lifestyle factors, and long-term QoL.

\section{Patients and Methods}

We conducted a cross-sectional anonymous online survey of Australian cancer survivors who had received neurotoxic chemotherapy as treatment for cancer. There was no exclusion regarding when participants completed treatment, and patients currently receiving chemotherapy were eligible to participate. Participants were

See JNCCN.org for supplemental online content. 
recruited between May 2016 and March 2019 via cancer research registers, social media, oncology waiting areas, and websites and newsletters of Australian cancer support and health advocacy organizations. Human research ethics approval was granted by the University of New South Wales Human Research Advisory Panel and the South Eastern Sydney Local Health District Human Research Ethics Committee.

The survey was developed using KeySurvey (WorldAPP; http://www.keysurvey.com). Participants were asked (1) whether they received chemotherapy that a health professional had indicated could cause tingling, numbness, or pins and needles in hands and feet, and (2) to select the chemotherapy they received from a list of neurotoxic agents. Participants were excluded if they answered "no" to the first question and stated that they had not received any chemotherapies listed; this combination indicated that they had not received neurotoxic chemotherapy treatment. Responses of excluded participants were not recorded by the survey platform.

\section{Survey Content}

The survey took approximately 35 minutes to complete, including demographics, cancer details, other cancerrelated AEs, and validated measures outlined as follows.

CIPN symptoms over the past week were assessed using the validated 11-item Functional Assessment of Cancer Therapy/Gynecologic Oncology Group - Neurotoxicity questionnaire [FACT/GOG-NTX], ${ }^{13}$ with scores ranging from 0 to 44 and higher scores indicating higher levels of symptoms. Neuropathic pain was assessed with the Douleur Neuropathique en 4 (DN4) questionnaire, ${ }^{14}$ with scores ranging from 0 to 7 and higher scores indicating worsening symptoms. An 11-point numeric pain rating scale (NPRS) $)^{15}$ assessed the most intense pain over the last 24 hours $(0=$ no pain; $10=$ worst possible pain $)$.

QoL was assessed using the validated RAND 36-Item Short Form Survey (SF-36) ${ }^{16}$ across 8 dimensions: physical functioning, bodily pain, role limitations due to physical health problems, personal or emotional problems, emotional well-being, social functioning, energy/fatigue, and general health. Subscale scores range from 0 to 100 , and total scores for the measure range from 0 to 800 , with higher scores indicating more optimal functioning. ${ }^{17}$

Comorbid health conditions were assessed using a list of conditions from the Self-Administered Comorbidity Questionnaire, ${ }^{18}$ including heart disease, high blood pressure, lung disease, diabetes, ulcer/stomach disease, kidney disease, liver disease, anemia/other blood disease, depression, osteoarthritis, rheumatoid arthritis, or back pain. A free-text field allowed the reporting of additional conditions.

The validated International Physical Activity Questionnaire-Short Form ${ }^{19}$ was used to assess physical activity during the past week in metabolic equivalent
(MET) minutes, via time spent performing low-, moderate-, and vigorous-intensity activity. Respondents who reported meeting Australian government guidelines for physical activity (600 MET-minutes/week, equivalent to 150 minutes of moderate intensity or 75 minutes of vigorous activity) ${ }^{20}$ were compared with those whose activity failed to meet the guidelines.

Respondents ranked the impact of treatment AEs on their lives, including fatigue, pain, neuropathy, nausea/ vomiting, anxiety, depression, insomnia, diarrhea, constipation, anemia, changes in sexual function, and infertility. A free-text field allowed the reporting of additional AEs. Respondents ranked as many AEs as were relevant, with 1 indicating the greatest impact.

\section{Statistical Analyses}

Statistical analyses were completed using SPSS Statistics version 23.0 (IBM Corp). Descriptive statistics were calculated for demographic and clinical characteristics. For the comparison of respondents reporting current CIPN symptoms to those without symptoms, $t$ tests were used for continuous variables and chi-square tests were used for categorical variables. Free-text responses were coded numerically such that they could be included in the analyses. Available case analyses were used, with pairwise deletion used to deal with missing data.

Analyses were conducted comparing respondents reporting high levels of CIPN symptoms (upper-tertile FACT/GOG-NTX; scoring $\geq 17$ ) and low levels of CIPN symptoms (lowest-tertile FACT/GOG-NTX; scoring $\leq 8$ ). Analyses also compared respondents who completed the survey within a year of finishing chemotherapy treatment with those who completed chemotherapy $\geq 6$ years earlier.

Multivariable linear regression analyses were performed to investigate associations between CIPN symptoms, clinical characteristics (years since completing chemotherapy, number of comorbid conditions, body mass index [BMI], general pain, burning pain), and sociodemographic characteristics (age, sex). Additional multivariable analyses were performed examining the relationships between QoL, CIPN, and physical activity, corrected for background variables including age, sex, years since completing chemotherapy, number of comorbid conditions, and BMI.

\section{Results}

\section{Cancer Survivor Demographic and}

Clinical Characteristics

Responses were received from 986 eligible respondents, with demographic and clinical characteristics presented in Table 1 and supplemental eTable 1 (available with this article at JNCCN.org). Results are presented as mean \pm standard deviation unless otherwise specified. 
Respondents had a mean age of $58 \pm 10.7$ years, and most were female ( $\mathrm{n}=820 ; 83.2 \%)$.

Breast cancer $(\mathrm{n}=581 ; 58.9 \%)$, colorectal cancer $(\mathrm{n}=133 ; 13.5 \%)$, and multiple myeloma $(\mathrm{n}=108 ; 11.0 \%)$ were the most commonly reported cancer types. Reported chemotherapies corresponded to treatments for these cancers: taxanes (docetaxel: $n=322$ [32.7\%]; paclitaxel: $\mathrm{n}=312$ [31.6\%]), platinum-based chemotherapies (oxaliplatin: $n=123$ [12.5\%]; cisplatin: $n=53$ [5.4\%]), thalidomide $(n=87 ; 8.8 \%)$, and bortezomib $(n=82 ; 8.3 \%)$.

One-quarter of respondents $(n=247 ; 25.1 \%)$ reported no comorbid health conditions, $30.1 \%(\mathrm{n}=297)$ reported 1 , $20.5 \%(n=202)$ reported 2 , and $24.3 \%(n=240)$ reported $\geq 3$. Comorbid conditions (supplemental eTable 1) included osteoarthritis $(n=264 ; 26.8 \%)$, back pain $(n=262$; $26.6 \%)$, high blood pressure $(n=226 ; 22.9 \%)$, depression $(\mathrm{n}=172 ; 17.4 \%)$, and diabetes $(\mathrm{n}=68 ; 6.9 \%)$.

\section{Burden of Neurotoxicity in Cancer Survivors}

Most respondents reported experiencing CIPN during $(\mathrm{n}=768 ; 77.9 \%)$ and after $(\mathrm{n}=785 ; 79.6 \%)$ chemotherapy. Most respondents $(\mathrm{n}=754 ; 76.5 \%)$ reported current CIPN, and $24.1 \%(n=238)$ reporting current symptoms indicated that these had not improved since chemotherapy completion. Participants reported experiencing CIPN symptoms for a duration of $3.6 \pm 3.5$ years.

More respondents with current CIPN reported symptoms affecting their lower limbs (feet: $n=689$ [91.3\%]; hands: $\mathrm{n}=566$ [75.1\%]). Accordingly, $28.7 \%$ $(\mathrm{n}=214)$ of respondents with current CIPN reported moderate-severe difficulties with walking, and $22.7 \%$ $(n=170)$ reported moderate-severe difficulties with hand function.

The validated FACT/GOG-NTX questionnaire captured differences in CIPN between respondents with and without current symptoms. Respondents reporting current CIPN had a mean score of $15.8 \pm 8.3$, whereas respondents without current CIPN had a mean score of $6.0 \pm 5.4(P<.001)$. Similarly, respondents with current CIPN had a mean DN4 score of $2.8 \pm 2.2$, and those without CIPN had a mean score of $0.8 \pm 1.3(P<.001)$. Respondents with current CIPN reported a mean NPRS score of $4.45 \pm 2.57$, and those without CIPN reported a mean score of $3.36 \pm 2.28$ $(P<.001)$. Each comparison indicated higher symptom levels in the current CIPN group.

Comparing female and male respondents, men were older (mean age, $63.0 \pm 12.6$ years vs $57.9 \pm 10.1$ years for women; $P<.001$ ) and reported a higher CIPN burden (FACT/GOG-NTX scores: $16.7 \pm 8.8$ vs $12.9 \pm 8.6$ for women; $P<.001$ ). No difference was observed in time since completing chemotherapy, number of comorbid conditions, burning pain, QoL, or BMI.

Participants ranked the impact of cancer treatment AEs on their lives. The largest number of respondents
Table 1. Demographic and Clinical Characteristics

\begin{tabular}{|c|c|}
\hline Characteristic & $\mathrm{n}(\%)$ \\
\hline Age, mean (SD), y & $58(10.7)$ \\
\hline $20-39$ & $41(4.2)$ \\
\hline $40-59$ & $447(45.3)$ \\
\hline $60-79$ & $479(48.6)$ \\
\hline$\geq 80$ & $13(1.3)$ \\
\hline Not reported & $6(0.6)$ \\
\hline \multicolumn{2}{|l|}{ Sex } \\
\hline Female & $820(83.2)$ \\
\hline Male & $163(16.5)$ \\
\hline \multicolumn{2}{|l|}{ Cancer type } \\
\hline Breast & $581(58.9)$ \\
\hline Colorectal & $133(13.5)$ \\
\hline Multiple myeloma & $108(11.0)$ \\
\hline Ovarian & $41(4.2)$ \\
\hline Lymphoma & $35(3.5)$ \\
\hline Other & $282(28.6)$ \\
\hline Years since completing treatment (SD) & $3.6(3.9)$ \\
\hline Currently on chemotherapy & $128(13.0)$ \\
\hline Completed chemotherapy $\leq 1$ y ago & $262(26.9)$ \\
\hline Completed chemotherapy $2-5$ y ago & $358(36.3)$ \\
\hline Completed chemotherapy $\geq 6$ y ago & $233(23.7)$ \\
\hline \multicolumn{2}{|l|}{ Stage at diagnosis } \\
\hline I & $160(16.2)$ \\
\hline II & $271(27.5)$ \\
\hline III & $341(31.8)$ \\
\hline IV & $85(8.6)$ \\
\hline Unknown & $153(15.5)$ \\
\hline \multicolumn{2}{|l|}{ Neurotoxic chemotherapy type } \\
\hline Docetaxel & $322(32.7)$ \\
\hline Paclitaxel & $312(31.6)$ \\
\hline Oxaliplatin & $123(12.5)$ \\
\hline Thalidomide & $87(8.8)$ \\
\hline Bortezomib & $82(8.3)$ \\
\hline Cisplatin & $53(5.4)$ \\
\hline Other & $113(11.5)$ \\
\hline Unknown & $102(10.3)$ \\
\hline \multicolumn{2}{|l|}{ Other cancer treatments received } \\
\hline Radiotherapy & $591(59.9)$ \\
\hline Surgery & $832(83.5)$ \\
\hline
\end{tabular}

rated fatigue as the $\mathrm{AE}$ with the greatest impact $(\mathrm{n}=423$; $42.9 \%$ rating it highest). CIPN was most commonly rated as having the second-greatest impact $(\mathrm{n}=270 ; 27.4 \%)$, followed by pain $(n=122 ; 12.4 \%)$, insomnia $(n=96 ; 9.7 \%)$, and changes in sexual function $(n=95 ; 9.6 \%)$. 
A minority of respondents had tried these treatment for CIPN ( $\mathrm{n}=168 ; 16.9 \%)$. Treatments included pregabalin $(\mathrm{n}=83 ; 8.4 \%)$, prescription/nonprescription analgesic medication $(n=65 ; 6.6 \%)$, exercise $(n=51 ; 5.2 \%)$, and acupuncture $(n=47 ; 4.7 \%)$. Low rates of treatment reflect the current lack of recommended treatments. Use of duloxetine, the only treatment with a moderate recommendation in the ASCO guidelines, ${ }^{21,22}$ was reported by $1.5 \%(n=15)$ of respondents.

\section{Long-Term Outcomes in Survivors With}

\section{Severe Neurotoxicity}

Comparing respondents reporting high (upper-tertile FACT/GOG-NTX) versus low (lowest-tertile FACT/ GOG-NTX) levels of CIPN symptoms, respondents who reported severe CIPN had poorer QoL scores on all SF-36 subscales $(P<.001$; Table 2, Figure 1A, supplemental eTable 2 ). Higher general and neuropathic pain were reported by respondents with high CIPN $(P<.001$; Figure $1 \mathrm{~B}, \mathrm{C})$. Respondents reporting severe CIPN were older $(P<.001)$, had a higher BMI $(P<.001$; Figure 1D), and had more comorbid conditions than those in the mild CIPN group $(P<.001$; Table 2). Respondents reporting severe CIPN completed chemotherapy more recently than those with mild CIPN $(P=.024)$. Respondents with severe CIPN more often reported receiving multiple neurotoxic chemotherapies $\left(\chi^{2}\right.$ $[1, \mathrm{n}=672]=5.5 ; P=.019)$ and were less likely to meet physical activity targets $\left(\chi^{2}[2, \mathrm{n}=672]=24.1 ; P<.001\right)$.

\section{Trajectory of Neurotoxicity}

Respondents who completed the survey $\leq 1$ year after completing chemotherapy $(\mathrm{n}=265 ; 26.9 \%)$ were younger and reported fewer comorbid conditions than those who completed the survey $\geq 6$ years after treatment completion $(\mathrm{n}=233$ [23.7\%]; $P<.001)$ (Table 3, Figure 2A, supplemental eTable 3). These groups did not differ in CIPN symptoms via FACT/GOG-NTX scores or pain levels via NPRS (Figure
2B, C). Of the respondents who completed chemotherapy within a year of taking the survey, $25.3 \%(n=65)$ reported no improvement in CIPN symptoms since finishing treatment, whereas $30.1 \%(\mathrm{n}=66)$ who completed treatment $\geq 6$ years earlier reported no improvement.

Compared with those who completed treatment recently, respondents who completed chemotherapy $\geq 6$ years earlier had better total QoL (Table 3, Figure 2D) and subscale scores assessing physical, emotional, and social functioning and energy (supplemental eTable 4).

\section{Physical Activity}

Most respondents self-reported meeting physical activity guidelines $(\mathrm{n}=787 ; 78.9 \%)$. These respondents showed better QoL across their total score $(P<.001$; Table 3$)$ and all subscales (supplemental eTable 5). Those meeting the guidelines reported a lower CIPN via FACT/GOG-NTX $(P<.001)$, lower BMI $(P<.001)$, longer duration of CIPN $(P=.009)$, and longer time since chemotherapy completion than those who did not meet the exercise guidelines $(P=.004)$. There was no difference between groups in age or number of comorbidities.

\section{Predictors of Long-Term Patient Outcomes}

Multivariable linear regression analyses were undertaken to identify predictors of severity of CIPN via FACT/GOGNTX $\left[F(7,874)=64.67 ; \quad P<.001 ; \quad R^{2}=0.34\right]$. Variables adding significantly to the predictors included pain (NPRS; $\beta=-0.36 ; \quad P<.001)$, burning pain $(\beta=0.25$; $P<.001$ ), sex (male sex associated with greater CIPN: $\beta=0.14 ; P<.001$ ), years since completing chemotherapy (shorter time associated with greater CIPN: $\beta=-0.10$; $P<.001)$, age $(\beta=0.80 ; P=.006)$, number of comorbid conditions ( $\beta=0.07 ; P=.02)$, and BMI $(\beta=0.07 ; P=.02)$.

In multivariable linear regression analyses on QoL scores $\left[F(7,903)=103.7 ; P<.001 ; R^{2}=0.45\right]$, significant predictor variables included comorbid conditions

Table 2. Outcomes and Characteristics in Respondents Reporting High Versus Low CIPN

\begin{tabular}{|c|c|c|c|c|c|}
\hline \multirow[b]{2}{*}{ Measure } & \multicolumn{2}{|c|}{ High CIPN } & \multicolumn{2}{|c|}{ Low CIPN } & \multirow[b]{2}{*}{$P$ Value } \\
\hline & $\mathrm{n}$ & Mean (SD) & $\mathrm{n}$ & Mean (SD) & \\
\hline Age & 333 & $59.6(10.8)$ & 335 & $56.4(10.3)$ & $<.001$ \\
\hline Years since completing chemotherapy & 326 & $3.2(3.1)$ & 325 & $3.8(4.0)$ & .024 \\
\hline General pain (NPRS; score range, 0-10) & 336 & $5.7(2.5)$ & 334 & $2.9(2.0)$ & $<.001$ \\
\hline BMI & 326 & $29.1(6.9)$ & 327 & $26.9(5.3)$ & $<.001$ \\
\hline
\end{tabular}

Abbreviations: BMI, body mass index; CIPN, chemotherapy-induced peripheral neurotoxicity; DN4, Douleur Neuropathique en 4; NPRS, numeric pain rating scale; QoL, quality of life; SF-36, RAND 36-Item Short-Form Survey. 
A

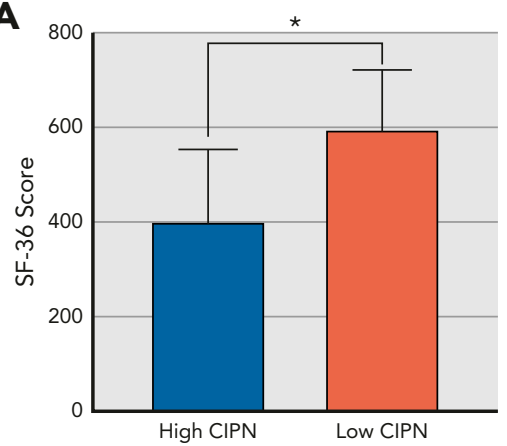

C

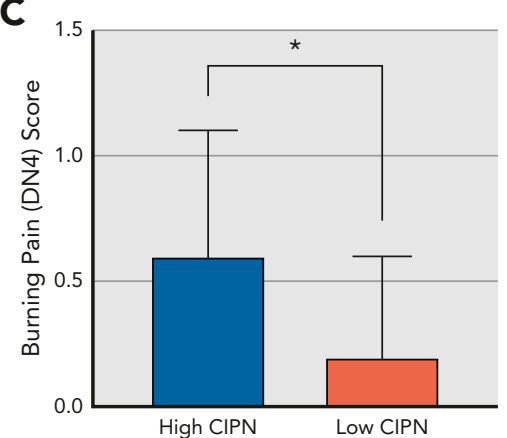

B

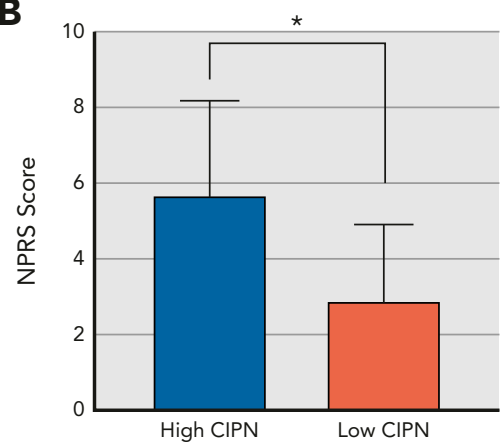

D

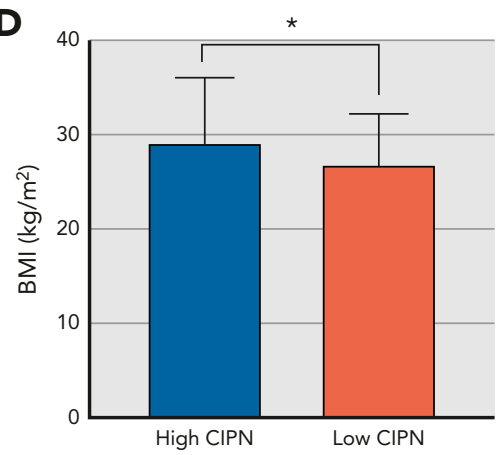

Figure 1. Effect of CIPN severity: (A) QoL score via SF-36, (B) pain level via NPRS, (C) burning pain level via DN4, and (D) BMI. Mean scores for each measure are presented with error bars representing standard deviations.

Abbreviations: CIPN, chemotherapy-induced peripheral neurotoxicity; DN4, Douleur Neuropathique en 4; NPRS, numeric pain rating scale; QoL, quality of life; SF-36, RAND 36-Item Short Form Survey.

*Significant difference, $P<.001$.

$(\beta=-33.19 ; P<.001)$, CIPN symptoms (FACT/GOG-NTX; $\beta=-8.76 ; \quad P<.001)$, years since completing chemotherapy $(\beta=5.75 ; P<.001)$, age $(\beta=3.13 ; P<.001)$, and physical activity $(\beta=0.01 ; P<.001)$.

\section{Discussion}

As one of the largest surveys focusing on the self-reported impact of CIPN in cancer survivors, our results reinforce the association of CIPN with lasting impacts across a range of domains. CIPN was reported by the majority of respondents and was associated with functional difficulties and reduced QoL. Multivariable analyses found associations between CIPN severity, pain, and comorbid health conditions. Although CIPN and pain severity persisted over time, respondents who completed chemotherapy a longer time beforehand reported better QoL.

Respondents experiencing high levels of CIPN symptoms more often reported receiving multiple neurotoxic chemotherapies, experiencing more comorbidities, and having a higher BMI than those with low CIPN. Although it is unclear whether higher BMI preceded the development of CIPN, CIPN was associated with multiple negative health impacts, potentially suggesting that respondents with severe CIPN experience poorer health

\section{Table 3. Outcomes and Characteristics Based on Adherence to Physical Activity Guidelines}

\begin{tabular}{|c|c|c|c|c|c|}
\hline \multirow[b]{2}{*}{ Measure } & \multicolumn{2}{|c|}{$\begin{array}{c}\text { Respondents } \\
\text { Meeting Guidelines }\end{array}$} & \multicolumn{2}{|c|}{$\begin{array}{l}\text { Respondents Not } \\
\text { Meeting Guidelines }\end{array}$} & \multirow[b]{2}{*}{$P$ Value } \\
\hline & n & Mean (SD) & n & Mean (SD) & \\
\hline CIPN burden (FACT/GOG-NTX total score; range, 0-44) & 782 & $12.7(8.3)$ & 153 & $16.4(9.4)$ & $<.001$ \\
\hline Years since completing chemotherapy & 764 & $3.7(4.0)$ & 143 & $2.7(3.5)$ & .004 \\
\hline Duration of CIPN symptoms (y) & 655 & $3.7(3.6)$ & 129 & $3.0(2.9)$ & .009 \\
\hline QoL (SF-36: total score; range, 0-800) & 787 & $524.9(152.6)$ & 153 & $407.4(163.3)$ & $<.001$ \\
\hline BMI & 762 & $27.1(5.9)$ & 150 & $29.8(6.2)$ & $<.001$ \\
\hline
\end{tabular}

Abbreviations: BMI, body mass index; CIPN, chemotherapy-induced peripheral neurotoxicity; FACT/GOG-NTX, Functional Assessment of Cancer Therapy/Gynecologic Oncology Group - Neurotoxicity; QoL, quality of life; SF-36, RAND 36-Item Short-Form Survey. 
A

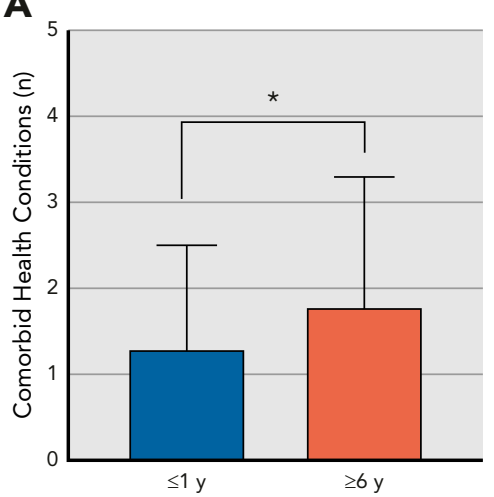

B

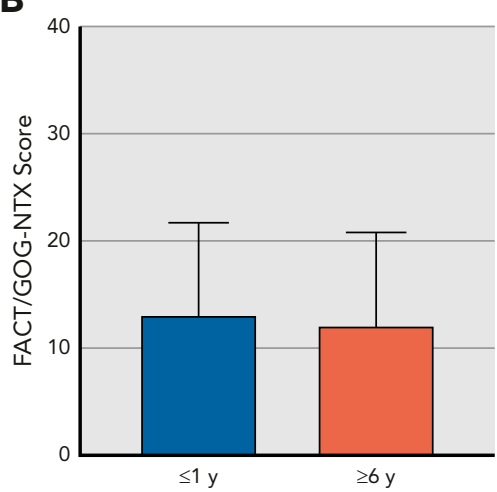

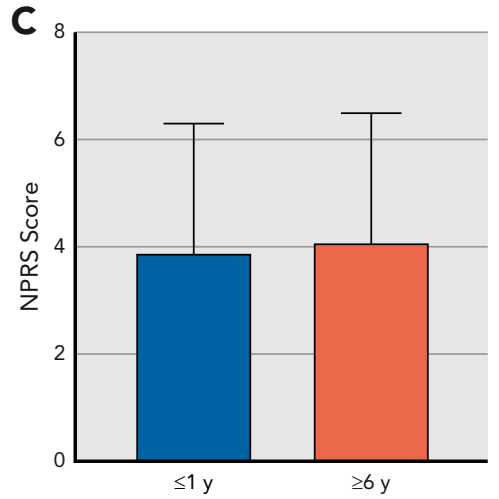

D

$E$
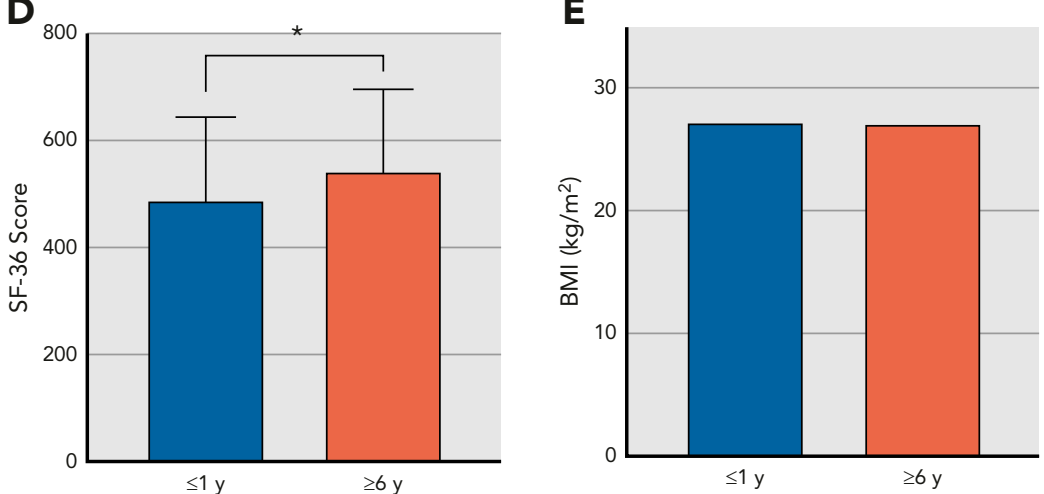

Figure 2. Symptom trajectory in respondents reporting CIPN: (A) number of comorbid health conditions, (B) FACT/GOG-NTX score, (C) pain level via NPRS, (D) QoL score via SF-36, and (E) BMI. Mean scores for each measure are presented with error bars representing standard deviations. Abbreviations: BMI, body mass index; CIPN, chemotherapy-induced peripheral neurotoxicity; FACT/GOG-NTX, Functional Assessment of Cancer Therapy/Gynecologic Oncology Group - Neurotoxicity; NPRS, numeric pain rating scale; QoL, quality of life; SF-36, RAND 36-Item Short Form Survey.

* Significant difference, $P<.001$

more generally. This suggestion is supported by previous research indicating poorer health in patients with CIPN, via greater symptom burden, comorbidities, and disability. ${ }^{3,9}$ Higher overall pain reported by respondents with severe CIPN highlights the need to better understand the relationship between pain and neuropathy.

CIPN and pain severity did not differ between respondents who completed chemotherapy recently and those who completed chemotherapy $\geq 6$ years earlier. Despite this finding, better QoL was reported by those who completed chemotherapy $\geq 6$ years earlier. CIPN symptom improvements may be more likely to occur soon after treatment, with symptom levels remaining stable for a proportion of patients with chronic symptoms. However, improvements in QoL with time suggest that a contributing factor may be adaptation to symptoms, with increasing resilience enabling improved QoL despite ongoing symptoms. Although adaptation to CIPN symptoms is challenging to empirically examine, qualitative interview studies have revealed potential coping and adaptive strategies that may be used by patients over time. . $3,24^{2}$

Further evidence of the relationship between general health, CIPN, and QoL was seen via multiple regression analyses, where predictors of CIPN severity included pain and the number of comorbid conditions. Similarly, the greatest predictors of QoL were the number of comorbid conditions and CIPN symptoms. Both factors predicted decreased QoL, further indicating the role of physical health in maintaining QoL in cancer survivors.

These results are in line with previous survey-based research finding CIPN to be a major complaint in cancer survivors. In a survey of 609 patients who received neurotoxic chemotherapy, $68.8 \%$ reported experiencing CIPN. ${ }^{3}$ An online survey found CIPN in one-third of 1,506 patients with breast cancer who received chemotherapy ${ }^{5}$ and in $78 \%$ of 1,360 women with ovarian cancer 2.3 years since completing chemotherapy. ${ }^{6}$ Similarly, $52 \%$ of 1,182 patients with acute myeloid leukemia reported CIPN 7.3 years postdiagnosis. ${ }^{4}$

Furthermore, our results support previous studies suggesting that CIPN has significant impacts on QoL. Surveys of survivors of colorectal cancer found decreased QoL in respondents with CIPN, ${ }^{7,8}$ and respondents with severe CIPN have been found to be more likely to experience anxiety and depression. ${ }^{25}$ In patients with ovarian cancer, those with CIPN have reported lower functioning and greater health-related worry. ${ }^{26}$ In a survey of patients 
with breast cancer, CIPN symptoms were considered a severe burden by two-thirds of the affected patients. ${ }^{5}$ In addition, a survey covering various cancer types found patients reporting neurotoxicity reported higher symptom burden and stress, and poorer QoL. ${ }^{3}$

Our study also suggests the positive effects of physical activity on CIPN, with respondents who met the physical activity guidelines reporting lower levels of CIPN. Respondents who reported meeting the guidelines also reported a longer time since completing chemotherapy. However, it is unclear whether this reduced CIPN resulted from exercise reducing their symptoms or from respondents with higher symptom levels being less able to exercise. Previous studies have found higher CIPN in respondents who do not meet recommended physical activity levels, ${ }^{8}$ and moderate-vigorous physical activity has been associated with lower CIPN in patients treated using taxanes. ${ }^{27} \mathrm{~A}$ number of studies have suggested a promising role for exercise in CIPN, ${ }^{28-30}$ and further research involving exercise programs for cancer survivors is required to clarify the relationship between physical activity and CIPN. Regardless, higher QoL and lower BMI of respondents meeting the activity guidelines indicate the positive effects of physical activity on well-being in cancer survivors.

A strength of our study is its size and the comprehensive nature of the measures used. Although patientreported outcomes are increasingly used to inform practice, ${ }^{31}$ this survey was self-reported and clinical details were not confirmed via medical records. Some respondents were not aware of the details of their diagnosis or treatment (eg, 10.3\% did not know the name of their chemotherapy and $15.5 \%$ did not know their cancer stage). Cancer survivors may have gaps in knowledge or recall about treatment details and may provide incorrect information..$^{32}$ This information gap could have contributed to selection bias because patients who were aware of treatment details or who remembered being informed of potential treatment AEs may have been less likely to have been screened out of the survey. Respondents who had accurate recall may have differed systematically from those who did not have accurate recall (previous research has found that accuracy of recall may decrease with age and vary across cancer types ${ }^{32}$ ), leading to potential differences in rates of survey completion between these 2 groups. Self-reported physical activity can also provide differing estimates to objective recording, ${ }^{33}$ tending to overestimate activity. ${ }^{34}$ Research using objective measures of activity is needed to confirm these results.

Participants were recruited primarily via cancer support organizations and may have differed systematically from less-engaged cancer survivors. In addition, individuals with current CIPN may have been more likely to respond than those without symptoms, potentially leading to selection bias. Accordingly, results must be interpreted with a high degree of caution regarding the representativeness of the sample, and inferences cannot be made regarding the overall population incidence or severity of CIPN. The largest respondent group was women with breast cancer, which was expected because breast cancer is one of the most commonly diagnosed cancers in Australia, with one of the highest survival rates ${ }^{35}$ among cancers treated using neurotoxic chemotherapy. However, this finding limited our ability to investigate the relative impact of different neurotoxic chemotherapies.

\section{Conclusions}

Results of our study indicate that CIPN symptoms are associated with impacts on QoL in the increasing population of cancer survivors. Respondents with severe CIPN were older and had a higher BMI and more comorbidities, suggesting reduced general health in this cohort. The association between physical activity and reduced CIPN symptoms warrants prospective examination to determine the role of exercise in symptom management. Although chronic CIPN may not reduce in severity over time, patients may adapt to chronic neuropathic symptoms and experience improved QoL. Understanding the risk factors related to persistent CIPN is important to enable the design of preventive strategies. There is a current lack of effective preventive measures for CIPN. Dose modification is the only preventive strategy recommended by ASCO guidelines, ${ }^{22}$ after the assessment of the risks and benefits of neurotoxic treatment (including consideration of conditions that may predispose a patient toward developing CIPN, such as diabetes or a family history of neuropathy). In addition, duloxetine is currently the only treatment for CIPN with a moderate recommendation. Other therapeutic management strategies have been trialed, including exercise, acupuncture, scrambler therapy, gabapentin or pregabalin, and topical gel treatments, but there is currently a lack of confirmatory evidence for their efficacy. ${ }^{22}$ Effective assessment, prevention, and treatment strategies are needed to improve QoL and decrease the considerable impact that CIPN has on the lives of cancer survivors.

\section{Acknowledgments}

Participants in this research were recruited via a number of cancer research registers. These included the Breast Cancer Network Australia Review and Survey Group, the Register4 cancer research register, and the Prostate Cancer Foundation of Australia's Pathfinder prostate cancer research register. We acknowledge the contribution of the members of these research registers who participated in this project. We also acknowledge the contribution of many other Australian cancer support and advocacy groups that promoted the survey and whose members participated in this project. 
Submitted November 30, 2020; final revision received February 10, 2021 . accepted for publication February 12, 2021.

Author contributions: Study concept and design: All authors. Data analysis and interpretation: Battaglini, Goldstein, Park. Statistical analysis: Battaglini. Writing - original draft: Battaglini. Writing - review and editing: All authors.

Disclosures: The authors have disclosed that they have not received any financial consideration from any person or organization to support the preparation, analysis, results, or discussion of this article.
Funding: This study was supported by a Cancer Institute NSW Program Grant (14/TPG/1-05) and a National Health and Medical Research Counci of Australia Project Grant (number 1080521). Dr. Park is supported by a National Health and Medical Research Council Career Development Fellowship (number 1148595)

Correspondence: Susanna B. Park, PhD, Brain and Mind Centre, The University of Sydney, 94 Mallett Street, Camperdown, NSW 2050, Australia. Email: susanna.park@sydney.edu.au

\section{References}

1. Miller KD, Siegel RL, Lin CC, et al. Cancer treatment and survivorship statistics, 2016. CA Cancer J Clin 2016;66:271-289.

2. Park SB, Goldstein D, Krishnan AV, et al. Chemotherapy-induced peripheral neurotoxicity: a critical analysis. CA Cancer J Clin 2013;63:419-437.

3. Miaskowski C, Mastick J, Paul SM, et al. Impact of chemotherapyinduced neurotoxicities on adult cancer survivors' symptom burden and quality of life. J Cancer Surviv 2018;12:234-245.

4. Crossnohere NL, Richardson DR, Reinhart C, et al. Side effects from acute myeloid leukemia treatment: results from a national survey. Curr Med Res Opin 2019;35:1965-1970.

5. Haidinger R, Bauerfeind I. Long-term side effects of adjuvant therapy in primary breast cancer patients: results of a web-based survey. Breast Care (Basel) 2019;14:111-116.

6. Webber K, Carolus E, Mileshkin L, et al. OVQUEST-life after the diagnosis and treatment of ovarian cancer-an international survey of symptoms and concerns in ovarian cancer survivors. Gynecol Oncol 2019;155:126-134.

7. Mols F, Beijers T, Lemmens V, et al. Chemotherapy-induced neuropathy and its association with quality of life among 2- to 11-year colorectal cancer survivors: results from the population-based PROFILES registry. J Clin Oncol 2013:31:2699-2707.

8. Mols F, Beijers AJM, Vreugdenhil G, et al. Chemotherapy-induced peripheral neuropathy, physical activity and health-related quality of life among colorectal cancer survivors from the PROFILES registry. J Cancer Surviv 2015;9:512-522.

9. Winters-Stone KM, Horak F, Jacobs PG, et al. Falls, functioning and disability among women with persistent symptoms of chemotherapy-induced peripheral neuropathy. J Clin Oncol 2017;35: 2604-2612.

10. Pachman DR, Qin R, Seisler DK, et al. Clinical course of oxaliplatininduced neuropathy: results from the randomized phase III trial N08CB (Alliance). J Clin Oncol 2015;33:3416-3422.

11. Shah A, Hoffman EM, Mauermann ML, et al. Incidence and disease burden of chemotherapy-induced peripheral neuropathy in a populationbased cohort. J Neurol Neurosurg Psychiatry 2018;89:636-641.

12. Pachman DR, Qin R, Seisler D, et al. Comparison of oxaliplatin and paclitaxel-induced neuropathy (Alliance A151505). Support Care Cancer 2016:24:5059-5068.

13 Cella D, Peterman A, Hudgens S, et al. Measuring the side effects of taxane therapy in oncology: the functional assessment of cancer therapytaxane (FACT-taxane). Cancer 2003;98:822-831.

14. Bouhassira $\mathrm{D}$, Attal $\mathrm{N}$, Alchaar $\mathrm{H}$, et al. Comparison of pain syndromes associated with nervous or somatic lesions and development of a new neuropathic pain diagnostic questionnaire (DN4). Pain 2005;114:29-36.

15. Downie WW, Leatham PA, Rhind VM, et al. Studies with pain rating scales. Ann Rheum Dis 1978;37:378-381.

16. Hays RD, Sherbourne CD, Mazel RM. The RAND 36-item health survey 1.0. Health Econ 1993;2:217-227.

17. Hays RD, Morales LS. The RAND-36 measure of health-related quality of life. Ann Med 2001;33:350-357.

18. Sangha O, Stucki G, Liang MH, et al. The Self-Administered Comorbidity Questionnaire: a new method to assess comorbidity for clinical and health services research. Arthritis Rheum 2003;49:156-163.

19. Craig CL, Marshall AL, Sjöström M, et al. International physical activity questionnaire: 12 -country reliability and validity. Med Sci Sports Exerc 2003;35:1381-1395.
20. Australian Government, Department of Health. Australia's physical activity and sedentary behaviour guidelines and the Australian 24-hour movement guidelines. Accessed December 11, 2019. Available at: https://www1.health.gov.au/internet/main/publishing.nsf/Content/healthpubhlth-strateg-phys-act-guidelines

21. Hershman $\mathrm{DL}$, Lacchetti $\mathrm{C}$, Dworkin $\mathrm{RH}$, et al. Prevention and management of chemotherapy-induced peripheral neuropathy in survivors of adult cancers: American Society of Clinical Oncology clinical practice guideline. J Clin Oncol 2014;32:1941-1967.

22. Loprinzi CL, Lacchetti C, Bleeker J, et al. Prevention and management of chemotherapy-induced neuropathy in survivors of adult cancers: ASCO guideline update. J Clin Oncol 2020;38:3325-3348.

23. Bakitas MA. Background noise: the experience of chemotherapy-induced peripheral neuropathy. Nurs Res 2007;56:323-331.

24. Drott J, Starkhammar H, Kjellgren K, et al. The trajectory of neurotoxic side effects' impact on daily life: a qualitative study. Support Care Cancer 2016;24:3455-3461.

25. Bonhof CS, van de Poll-Franse LV, Vissers PAJ, et al. Anxiety and depression mediate the association between chemotherapy-induced periphera neuropathy and fatigue: results from the population-based PROFILES registry. Psychooncology 2019;28:1926-1933.

26. Ezendam NPM, Pijlman B, Bhugwandass C, et al. Chemotherapy-induced peripheral neuropathy and its impact on health-related quality of life among ovarian cancer survivors: results from the population-based PROFILES registry. Gynecol Oncol 2014;135:510-517.

27. Greenlee $\mathrm{H}$, Hershman DL, Shi Z, et al. BMI, lifestyle factors and taxaneinduced neuropathy in breast cancer patients: the Pathways Study. J Nat Cancer Inst 2016;109:djw206.

28. Streckmann F, Kneis S, Leifert JA, et al. Exercise program improves therapy-related side-effects and quality of life in lymphoma patients undergoing therapy. Ann Oncol 2014;25:493-499.

29. Zimmer $P$, Trebing $S$, Timmers-Trebing $U$, et al. Eight-week, multimoda exercise counteracts a progress of chemotherapy-induced peripheral neuropathy and improves balance and strength in metastasized colorectal cancer patients: a randomized controlled trial. Support Care Cancer 2018;26:615-624.

30. McCrary JM, Goldstein D, Sandler CX, et al. Exercise-based rehabilitation for cancer survivors with chemotherapy-induced peripheral neuropathy. Support Care Cancer 2019;27:3849-3857.

31. Calvert MJ, O'Connor DJ, Basch EM. Harnessing the patient voice in real-world evidence: the essential role of patient-reported outcomes. Nat Rev Drug Discov 2019;18:731-732.

32. Nissen MJ, Tsai ML, Blaes AH, et al. Breast and colorectal cancer survivors' knowledge about their diagnosis and treatment. J Cancer Surviv 2012;6:20-32.

33. Prince SA, Adamo KB, Hamel ME, et al. A comparison of direct versus self-report measures for assessing physical activity in adults: a systematic review. Int J Behav Nutr Phys Act 2008;5:56.

34. Lee PH, Macfarlane DJ, Lam TH, et al. Validity of the International Physical Activity Questionnaire Short Form (IPAQ-SF): a systematic review. Int J Behav Nutr Phys Act 2011;8:115.

35. Australian Institute of Health and Welfare. Cancer in Australia 2019 Cancer series number 119. Catalog number CAN 123. Accessed

February 18, 2021. Available at: https://www.aihw.gov.au/ getmedia/8c9fcf52-0055-41a0-96d9-f81b0feb98cf/aihw-can-123. pdf.aspx?inline $=$ true 
Supplemental online content for:

\section{Chemotherapy-Induced Peripheral Neurotoxicity in Cancer Survivors: Predictors of Long-Term Patient Outcomes}

Eva Battaglini, PhD; David Goldstein, MBBS; Peter Grimison, PhD, MBBS; Susan McCullough;

Phil Mendoza-Jones; and Susanna B. Park, PhD

J Natl Compr Canc Netw 2021;19(7):821-828

eTable 1: Demographic Characteristics

eTable 2: Outcomes and Characteristics in Respondents Reporting High Versus Low CIPN

eTable 3: Outcomes and Characteristics in Respondents $\leq 1$ and $\geq 6$ Years Posttreatment

eTable 4: Outcomes and Characteristics in Respondents $\leq 1$ and $\geq 6$ Years Posttreatment via SF-36

eTable 5: Outcomes and Characteristics in Respondents Based on Adherence to Physical Activity Guidelines 


\section{eTable 1. Demographic Characteristics}

Characteristic

n (\%)

Education level

\begin{tabular}{lc} 
No school certificate & $13(1.3)$ \\
\hline Secondary school & $179(18.2)$ \\
\hline Trade/Apprenticeship & $49(5)$ \\
\hline Vocational college & $235(23.8)$ \\
\hline Bachelor's degree & $293(29.7)$ \\
\hline Postgraduate degree & $215(21.8)$
\end{tabular}

Marital status

\begin{tabular}{lc}
\hline Never married & $86(8.7)$ \\
\hline Married/de Facto & $733(74.3)$ \\
\hline Divorced/Separated & $122(12.4)$ \\
\hline Widowed & $40(4.1)$
\end{tabular}

Current employment status

\begin{tabular}{lc}
\hline Full time & $193(19.6)$ \\
\hline Part time & $231(23.4)$ \\
\hline Studying & $9(0.9)$ \\
\hline Sick leave & $37(3.8)$ \\
\hline Retired & $364(36.9)$ \\
\hline Not working due to disability & $69(7)$ \\
\hline Not employed, looking for work & $22(2.2)$ \\
\hline Not employed, not looking for work & $43(4.4)$
\end{tabular}

Language spoken at home

\begin{tabular}{lc}
\hline English only & $912(92.5)$ \\
\hline Other & $62(6.3)$
\end{tabular}

Comorbid health conditions

\begin{tabular}{lc}
\hline Osteoarthritis & $264(26.8)$ \\
\hline Back pain & $262(26.6)$ \\
\hline High blood pressure & $226(22.9)$ \\
\hline Depression & $172(17.4)$ \\
\hline Diabetes & $68(6.9)$ \\
\hline Anemia & $65(6.6)$ \\
\hline Heart disease & $60(6.1)$ \\
\hline Rheumatoid arthritis & $36(3.7)$ \\
\hline Thyroid disorder & $35(3.5)$ \\
\hline Liver disease & $26(2.6)$ \\
\hline Lung disease & $26(2.6)$ \\
\hline High cholesterol & $25(2.5)$ \\
\hline Kidney disease & $23(2.3)$ \\
\hline Ulcer or stomach disease & $21(2.1)$ \\
\hline
\end{tabular}




\begin{tabular}{|c|c|c|c|c|c|}
\hline \multirow[b]{2}{*}{ SF-36 Measure } & \multicolumn{2}{|c|}{$\begin{array}{c}\text { Respondents Reporting } \\
\text { High CIPN }\end{array}$} & \multicolumn{2}{|c|}{$\begin{array}{c}\text { Respondents Reporting } \\
\text { Low CIPN }\end{array}$} & \multirow[b]{2}{*}{$P$ Value } \\
\hline & $\mathrm{n}$ & Mean (SD) & n & Mean (SD) & \\
\hline Physical functioning & 337 & $54.6(24.7)$ & 335 & $82.3(18.2)$ & $<.001$ \\
\hline Role limitations due to physical health & 334 & $29.3(36.2)$ & 334 & $73.1(37.6)$ & $<.001$ \\
\hline Role limitations due to emotional problems & 336 & $55.4(42.4)$ & 335 & $81.0(31.5)$ & $<.001$ \\
\hline Energy/Fatigue & 337 & $34.5(20.4)$ & 334 & $55.1(19.2)$ & $<.001$ \\
\hline Emotional well-being & 337 & $66.3(19.2)$ & 334 & $76.7(16.0)$ & $<.001$ \\
\hline Social functioning & 337 & $65.4(24.3)$ & 335 & $84.5(18.4)$ & $<.001$ \\
\hline Pain & 337 & $51.2(25.2)$ & 335 & $77.1(19.6)$ & $<.001$ \\
\hline General health & 337 & $45.1(22.3)$ & 335 & $65.9(19.8)$ & $<.001$ \\
\hline
\end{tabular}

Abbreviations: CIPN, chemotherapy-induced peripheral neurotoxicity; SF-36, RAND 36-Item Short-Form Survey (subscale score range, 0-100).

\begin{tabular}{|c|c|c|c|c|c|}
\hline \multirow[b]{2}{*}{ Measure } & \multicolumn{2}{|c|}{$\leq 1$ Year Posttreatment } & \multicolumn{2}{|c|}{$\geq 6$ Years Posttreatment } & \multirow[b]{2}{*}{$P$ Value } \\
\hline & $\mathrm{n}$ & Mean (SD) & $\mathbf{n}$ & Mean (SD) & \\
\hline CIPN burden (FACT/GOG-NTX total score; range, 0-44) & 263 & $13.2(8.5)$ & 233 & $12.2(8.6)$ & .19 \\
\hline Age & 264 & $55.9(11.4)$ & 231 & $61.0(10.1)$ & $<.001$ \\
\hline Number of comorbid health conditions & 265 & $1.3(1.2)$ & 233 & $1.8(1.5)$ & $<.001$ \\
\hline General pain (NPRS; score range, $0-10$ ) & 265 & $3.9(2.4)$ & 231 & $4.1(2.4)$ & .36 \\
\hline Burning pain (DN4 item 1) & 245 & $0.3(0.5)$ & 221 & $0.4(0.5)$ & .04 \\
\hline QoL (SF-36: total score; range, $0-800$ ) & 265 & $490.6(154.1)$ & 233 & $544.0(153.1)$ & $<.001$ \\
\hline BMI & 254 & $27.2(5.5)$ & 227 & $27.1(5.2)$ & .77 \\
\hline
\end{tabular}

Abbreviations: BMI, body mass index; CIPN, chemotherapy-induced peripheral neurotoxicity; DN4, Douleur Neuropathique en 4; FACT/GOG-NTX, Functional Assessment of Cancer Therapy/Gynecologic Oncology Group - Neurotoxicity; NPRS, numeric pain rating scale; QoL, quality of life; SF-36, RAND 36-Item Short-Form Survey.

\begin{tabular}{|c|c|c|c|c|c|}
\hline SF-36 Measure & $n$ & Mean (SD) & $\mathrm{n}$ & Mean (SD) & $P$ Value \\
\hline Physical functioning & 265 & $68.0(25.2)$ & 233 & $73.0(23.2)$ & .02 \\
\hline Role limitations due to physical health & 265 & $46.5(43.5)$ & 231 & $64.5(40.2)$ & $<.001$ \\
\hline Role limitations due to emotional problems & 264 & $64.6(40.2)$ & 233 & $76.0(36.3)$ & .001 \\
\hline Emotional well-being & 264 & $69.5(18.5)$ & 232 & $74.8(17.2)$ & .001 \\
\hline Social functioning & 265 & $73.5(21.8)$ & 233 & $81.8(22.4)$ & $<.001$ \\
\hline Pain & 265 & $67.7(23.9)$ & 233 & $66.2(23.5)$ & .49 \\
\hline General health & 265 & $57.1(22.1)$ & 233 & $58.6(22.7)$ & .47 \\
\hline
\end{tabular}

Abbreviation: SF-36, RAND 36-Item Short-Form Survey (subscale score range, 0-100). 


\section{eTable 5. Outcomes and Characteristics in Respondents Based on Adherence to Physical} Activity Guidelines

\begin{tabular}{|c|c|c|c|c|c|}
\hline \multirow[b]{2}{*}{ SF-36 Measure } & \multicolumn{2}{|c|}{$\begin{array}{c}\text { Respondents } \\
\text { Meeting Guidelines }\end{array}$} & \multicolumn{2}{|c|}{$\begin{array}{l}\text { Respondents Not } \\
\text { Meeting Guidelines }\end{array}$} & \multirow[b]{2}{*}{$P$ Value } \\
\hline & n & Mean (SD) & n & Mean (SD) & \\
\hline Physical functioning & 787 & $72.9(22.2)$ & 153 & $48.7(28.6)$ & $<.001$ \\
\hline Role limitations due to physical health & 784 & $56.5(42.0)$ & 152 & $30.3(40.2)$ & $<.001$ \\
\hline Role limitations due to emotional problems & 785 & $71.5(38.0)$ & 153 & $59.3(42.6)$ & .001 \\
\hline Energy/Fatigue & 787 & $47.8(21.3)$ & 151 & $33.2(20.5)$ & $<.001$ \\
\hline Emotional well-being & 786 & $72.8(17.6)$ & 151 & $68.2(18.2)$ & .005 \\
\hline Social functioning & 787 & $78.6(21.5)$ & 153 & $65.7(25.6)$ & $<.001$ \\
\hline Pain & 787 & $67.4(23.6)$ & 153 & $57.2(27.6)$ & $<.001$ \\
\hline General health & 787 & $57.6(22.4)$ & 153 & $43.8(23.2)$ & $<.001$ \\
\hline
\end{tabular}

Abbreviation: SF-36, RAND 36-Item Short-Form Survey (subscale score range, 0-100). 